\title{
REICHL, K., Edige, A Karakalpak Oral Epic - as Performed by Jumabay Bazarov
}

Helsinki, Suomalainen Tiedeakatemia Academia Scientiarum Fennica, 2007, 498 p. ISSN 0014-5815 ISBN (hard) 978-951-41-1012-2. ISBN (soft) 978-951-41-1013-9

\section{Frédéric Léotar}

\section{OpenEdition}

\section{Journals}

Édition électronique

URL : https://journals.openedition.org/emscat/1647

DOI : 10.4000/emscat.1647

ISSN : 2101-0013

Éditeur

Centre d'Etudes Mongoles \& Sibériennes / École Pratique des Hautes Études

\section{Référence électronique}

Frédéric Léotar, « REICHL, K., Edige, A Karakalpak Oral Epic - as Performed by Jumabay Bazarov », Études mongoles et sibériennes, centrasiatiques et tibétaines [En ligne], 40 | 2009, mis en ligne le 28 décembre 2009, consulté le 13 juillet 2021. URL : http://journals.openedition.org/emscat/1647 ; DOI : https://doi.org/10.4000/emscat.1647

Ce document a été généré automatiquement le 13 juillet 2021.

(c) Tous droits réservés 


\section{REICHL, K., Edige, A Karakalpak Oral Epic - as Performed by Jumabay Bazarov}

Helsinki, Suomalainen Tiedeakatemia Academia Scientiarum Fennica, 2007, 498 p. ISSN 0014-5815 ISBN (hard) 978-951-41-1012-2. ISBN (soft) 978-951-41-1013-9

Frédéric Léotar

\section{RÉFÉRENCE}

REICHL, K., Edige, A Karakalpak Oral Epic - as Performed by Jumabay Bazarov, Suomalainen Tiedeakatemia Academia Scientiarum Fennica,(Helsinki 2007), 498 p.

1 L'ouvrage de Karl Reichl consacré à l'épopée karakalpake Edige constitue une avancée importante dans l'étude des épopées centrasiatiques. En effet, cette édition fournit non seulement la traduction intégrale en anglais d'une épopée karakalpake d'importance (précédée du texte original) mais elle en offre aussi une analyse détaillée, menée à différents niveaux. Le tout est complété par des documents audio-vidéo montrant quelques extraits significatifs de l'exécution.

2 Edige fait partie d'un riche fond d'épopées héroïques (dastan) répertoriées chez les Karakalpaks. Ce peuple turcophone, de tradition semi-nomade, islamisé, vit dans les zones désertiques d'Ouzbékistan, entre le Kazakhstan et le Turkménistan. Les enregistrements d'Edige par K. Reichl (1986 et 1993) ont été réalisés auprès de Jumabay Bazarov (Jumabay-jïraw), vraisemblablement le dernier barde connaissant l'épopée dans son intégralité. C'est dire à quel point la transmission des épopées s'est tarie, leur exécution se limitant aujourd'hui à des extraits relativement courts en comparaison du fonds d'archives référencées à l'Académie des Sciences d'Ouzbékistan (branche karakalpake). 
3 Rappelons en premier lieu la difficulté inhérente à la traduction d'une épopée transmise oralement, de génération en génération, et inspirée de faits historiques remontant au $\mathrm{XV}^{\mathrm{e}}$ siècle. Mais $\mathrm{K}$. Reichl, au-delà de sa profonde connaissance des épopées centrasiatiques, a pu compter dans cette entreprise sur l'important éclairage fourni par Jumabay-Jiraw. À cela s'ajoute la collaboration de l'auteur avec plusieurs spécialistes karakalpaks, parmi lesquels l'ethnographe T. Qanaatov, reconnu pour sa profonde connaissance des formules dialectales, des archaïsmes et des emprunts de la langue karakalpake au kazakh.

4 Les six premiers chapitres de l'ouvrage sont une mise en contexte de l'épopée selon différentes perspectives. Le chapitre 1 donne un aperçu de la situation pré-soviétique et contemporaine des Karakalpaks ainsi que de l'épopée en tant que telle. Y sont abordés deux thèmes chers à l'auteur: la tension générée dans l'épopée entre des éléments historiques et des éléments mythiques (que l'on retrouve dans bon nombre de récits transmis oralement), et la légitimité à considérer Edige, ainsi que les autres dastan, comme des oral epics même si le texte n'y est pas intégralement versifié.

5 Le chapitre 2, The Edige of History, est plus spécifiquement consacré à la dimension historique du personnage Edige, un émir du clan Mangghyt cité dans les sources russes pour avoir assiégé et rançonné Moscou en 1408. L'importance historique qui lui est accordée repose sur son rôle de fondateur des Noghay, un khanat dans lequel s'est dissoute la Horde d'or au XVe siècle. En effet, les Karakalpaks, dont l'ethnonyme apparaît au $\mathrm{XVl} \mathrm{l}^{\mathrm{e}}$ siècle, considèrent les Noghay comme leurs ancêtres. Les trois personnages historiques sur lesquels repose l'épopée sont Edige (le héros éponyme), Tokhtamysh considéré historiquement comme le dernier khan important de la Horde d'Or (d'origine mongole mais rapidement turcisée et islamisée), et Sätemir (le fameux Tamerlan), auquel se ralliera Edige contre Tokhtamysh.

6 Le chapitre suivant (chapitre 3) nous plonge dans différentes versions turciques d'Edige : en noghay, kazakh, tatar et bashkir. L'auteur s'appuie sur les différentes versions connues, qu'il résume et classe selon leur appartenance ethnique. De leur comparaison thématique se dégage un certain nombre de similarités et de différences, au niveau de la généalogie et des rapports entre les personnages principaux ainsi que de leur rôle dans l'épopée. À la fin du chapitre (pp.49-50), l'auteur procède à un récapitulatif appréciable de la trame commune à toutes ces versions, en quatorze points.

7 Le chapitre 4 traite des versions karakalpakes de l'épopée Edige. Après une présentation succincte des onze versions recueillies précédemment au cours $\mathrm{du} \mathrm{Xx}^{\mathrm{e}}$ siècle, l'auteur introduit le barde Jumabay-jïraw et la version qu'il a enregistrée auprès de lui en 1993 (la fin étant complétée par une version datant de 1986). L'action de l'épopée est ensuite résumée en cinquante et une parties et comparée à la plus ancienne version (datant de 1903, et enregistrée auprès de Bekimbet-jïraw). La grande homogénéité (constancy) qui en découle en termes d'actions, de descriptions de scènes ainsi que d'une terminologie très proche entre les deux versions, incite l'auteur à aborder, dans le chapitre suivant, la question de la transmission.

8 Dans le chapitre 5, Reichl explore tout d'abord la piste des qissa-xan, ces « liseurs de contes » qui récitent des textes appris par cœur. L'auteur cherche ainsi à déterminer si ce type de pratique où la tradition orale ou "orature ", pour reprendre l'expression utilisée par Rémy Dor, est figée par l'écriture avant d'être transmise favorise une homogénéisation thématique. En effet, les qissa-xan mettaient par écrit toutes sortes de 
récits, dont des épopées héroïques, qu'ils transmettaient ensuite à la population illettrée. (Précisons que plusieurs écoles de qissa-xan ont été fondées dès la fin du XIX siècle et qu'au-delà des épopées, elles avaient pour objectif premier d'instruire le peuple à la littérature arabo-persane, voir Pahratdinov 2002). Mais l'auteur privilégie plutôt la force de la transmission au sein d'une même généalogie (p. 83) et un entraînement intensif des bardes pour expliquer le caractère relativement figé de l'épopée. Sa démonstration, basée sur la comparaison de trois scènes issues de cinq versions différentes est, de ce point de vue, éloquente (voir le texte original).

Dans le chapitre 6, l'auteur cherche le prototype thématique (stemma) d'Edige à travers ses différentes versions, une entreprise limitée par les textes disponibles qui ne permettent pas de remonter au-delà du XIXe siècle. Alors, plutôt que de saisir la forme épique dans son ensemble, Reichl propose de sélectionner exclusivement les éléments communs à toutes les versions karakalpakes, postulant que ces similarités renvoient à un même schéma, modelé à l'époque où il a été vraisemblablement conçu (XIVe- XVI siècles). En effet, il semble peu probable que ces éléments aient été ajoutés ultérieurement et ce de façon uniforme dans toutes les versions. L'auteur choisit de se concentrer sur deux personnages pour illustrer sa démonstration : Baba Tükli, ancêtre du héros, et Sïpïra-jüraw, conseiller âgé du khan Tokhtamysh. Le premier fait figure de propagateur de l'Islam auprès des khans de la Horde d'Or, bien qu'il ait des pouvoirs typiquement chamaniques (renvoyant à la période pré-islamique). Le second, généalogiste et prophète, représente l'ancêtre supposé des bardes karakalpaks.

Les trois chapitres suivants (chapitres 7-9) sont d'ordre analytique et méthodologique. En cela, ils introduisent plus précisément les différents niveaux structurels de l'épopée (texte, performance, musique).

11 Le chapitre 7 est consacré à la structure métrique du texte. L'auteur y rappelle les caractéristiques du karakalpak ayant des incidences d'ordre structurel (langue agglutinante avec harmonie vocalique) et procède à une analyse stylistique sommaire du texte, tout en rappelant qu'une étude poétique ne peut être complète sans la prise en compte de sa dimension musicale (sections chantées).

La question des caractéristiques performatives du texte chanté est développée dans le chapitre 8, consacré aux critères sélectionnés par l'auteur (linguistiques, musicaux, kinésiques) afin d'aboutir à une édition de l'épopée qui ne soit pas limitée à sa seule dimension littéraire (voir en particulier la section 8.1 From Word to Text).

Dans le chapitre 9 , Reichl procède à une analyse musicologique synthétique des parties exclusivement chantées. Après avoir classifié les techniques expressives utilisées par le barde Jumabay-jïrawlors de son exécution (narrating, declaiming, narratingdeclaiming, singing, singing in a recitative, p. 166), l'auteur se concentre sur l'aspect mélodico-rythmique des passages chantés. L'analyse lui permet de dégager quatre mélodies fondamentales (mélodies A, B, C, D). Il est remarquable de voir ici que l'analyse musicologique est traitée avec autant d'attention par l'auteur que l'analyse poétique exposée au chapitre $7 \mathrm{avec}$, ligne après ligne, une description de la transformation thématique opérée par le barde. Il en résulte une transcription de chaque thème musical dans sa plus simple expression, suivie de ses principales variations mélodico-rythmiques, et enfin de son modèle mélodique (melodic pattern). En conclusion, l'auteur tente de démontrer que la répétition de quatre mélodies sur un récit de 27600 mots n'est pas monotone puisqu'un principe de variation en termes expressifs et mélodico-rythmiques a lieu d'une ligne à l'autre (pp. 176-178). 

seconde moitié de l'ouvrage. Ici, nous soulignerons les soins apportés par l'auteur pour intégrer dans la mise par écrit du récit épique, sa dimension musicale à travers des symboles (renvoyant aux mélodies jouées en solo à la vièle qobïz [kobyz]) et la transcription sur portée des principaux thèmes chantés. À cela s'ajoutent des notes relatives aux différents aspects linguistiques du texte (pp. 432-455) et un commentaire sur la dimension sémantique de certains passages qui prend en considération cinq autres versions d'Edige (pp. 456-478).

15 Concernant la conclusion du chapitre 9, nous aimerions apporter deux remarques. Car, comme nous venons de le voir, l'analyse musicologique de tout le chapitre a démontré qu'en deçà d'un certain niveau de variations pour le moins mineures, la musique vocale repose sur quatre mélodies de base. En fait, l'analyse menée par l'auteur permet d'attester que les mélodies $\mathrm{A}$ et $\mathrm{D}$ sont clairement apparentées, l'une dérivant probablement de l'autre, ce qui réduit en définitive le nombre de thèmes musicaux à trois.

Or, la question d'une éventuelle "monotonie» du matériau mélodico-rythmique concerne plus le pôle de la réceptivité (l'esthésique en termes sémiologiques), que celui de l'analyse musicologique proprement dite. En d'autres termes, plus que l'analyse externe, le témoignage des auditeurs locaux demeure de toute première importance dans l'évaluation de critères esthétiques. Ces derniers renvoient aux jugements de goût et à la relation entre barde et public qui doit aussi influencer l'exécution de l'épopée (comme elle a nécessairement influencé cette version).

De plus, la «monotonie» est établie ici selon une conception de la musique où la variation est une qualité nécessaire. Or, il semble bien que, à côté des normes poétiques, performatives et musicales établies par l'auteur, la répétition de cellules mélodico-rythmiques ait aussi un rôle structurant. Les caractéristiques musicologiques données par Reichl: "Syllabic style, stichic melodies, a comparatively small melodic ambitus, accompaniment by a string instrument and a predilection for melodies circling, as in a recitative, around one or two notes...» (p. 176) sont, en Asie centrale, globalement valables en dehors des épopées héroïques. En effet, l'ensemble des musiques populaires répond parfaitement à de tels critères. Or, la répétition cyclique a une place importante dans les musiques à caractère religieux, sacré, thérapeutique, fonctionnel. Ici, ce n'est pas la diversité mélodico-rythmique qui importe mais plutôt la répétition d'une même cellule intégrant de légères variations (écouter Léotar 2008 : pl. 21-23). Le retour au modèle, sans cesse répété, et sans cesse légèrement varié, donne ainsi toute sa force à la mélodie, qu'elle soit chantée pour endormir un enfant, soigner un malade, traire une vache ou encore, probablement, pour transmettre un récit épique... Mais le traitement de ces deux thématiques aurait débordé certainement le cadre de cet ouvrage, sans compter le déclin des traditions épiques karakalpakes et la réduction des épopées dans la sphère sociale à un simple et court intermède.

En conclusion, cette édition rend un très bel hommage à une épopée aujourd'hui à l'état de vestige chez les Karakalpaks. Elle représente ainsi un document d'importance pour l'histoire et la mythologie de ce peuple. Elle apporte en outre un éclairage sur de nombreux aspects liés au texte mais aussi, et c'est tout le défi de cet ouvrage, à l'histoire et à la culture karakalpakes, aux dimensions musicales et performatives de l'épopée. Plus qu'introductif, le traitement de ces différents aspects donne une compréhension approfondie de l'épopée et démontre à quel point elle est inscrite

Études mongoles et sibériennes, centrasiatiques et tibétaines, 40 | 2009 
culturellement dans un tout d'où le récit, en tant que texte, ne constitue qu'une partie. Enfin, les résultats analytiques auxquels l'auteur a abouti, et dont nombre d'entre eux sont plus largement partagés au sein des sociétés turciques, constituent un apport précieux pour le développement des études comparatives dans la région.

\section{BIBLIOGRAPHIE}

Léotar, F.

2008 Karakalpakistan - La Voix des Ancêtres, Buda Records (CD, DVD, fichier pdf et livret de 26 p.).

Nattiez, J.-J.

Musicologie générale et sémiologie (Paris : Christian Bourgois éditeur).

Pahratdinov K. A.

2002 Puti vozniknovenija i formirovanija škol karakalpakskih kyssahanov-poètov v konce XIX $i v$ načale XX vekov. [Storytelling in Karakalpak Literature of the end of 19th - beginning of 20th centuries], 2002, Governmental Karakalpak University, summary of thesis (avtoreferat : UDK-894.375), short summary in Karakalpak \& English, 23p.

Reichl, K.

1992 Turkic Oral Epic Poetry : Traditions, Forms, Poetic Structure (New-York \& London: Garland Publ.). 2001 L'épopée orale turque d'Asie centrale. Contes épiques nanaïs, Études mongoles et sibériennes, centrasiatiques et tibétaines, $n^{\circ} 32$.

\section{AUTEURS}

\section{FRÉDÉRIC LÉOTAR}

